\title{
Optimal Power Flow operation of an Interline Current Flow Controller in an hybrid AC/DC meshed grid
}

\author{
Joan Sau-Bassols ${ }^{\mathrm{a}}$, Quanyu Zhao ${ }^{\mathrm{b}}$, Javier García-González ${ }^{\mathrm{b}}$, Eduardo Prieto-Araujo ${ }^{\mathrm{a}}$, \\ Oriol Gomis-Bellmunt ${ }^{\mathrm{a}}$ \\ ${ }^{a}$ Centre d'Innovació Tecnològica en Convertidors Estàtics i Accionaments (CITCEA-UPC), \\ Departament d'Enginyeria Elèctrica, Universitat Politècnica de Catalunya. ETS d'Enginyeria \\ Industrial de Barcelona, Av. Diagonal,647, Pl. 2.08028 Barcelona, Spain (joan.sau@citcea.upc.edu) \\ ${ }^{b}$ Institute for Research in Technology (IIT), ICAI School of Engineering, Comillas Pontificial \\ University, Madrid, Spain (quanyu.zhao23@gmail.com)
}

\begin{abstract}
This paper is meant to show that an interline Current Flow Controller (CFC) is able to reduce the operating costs of hybrid $\mathrm{AC} / \mathrm{DC}$ meshed grids by alleviating the congestion within the DC lines. The work considers a unidirectional DC/DC CFC with a simple structure and its average model is introduced into the mathematical formulation of the Optimal Power Flow (OPF) problem of an hybrid AC/DC meshed grid, which also considers the losses of the Voltage Source Converters (VSC). The CFC restrictions and the different connection arrangements of the device into the $\mathrm{AC} / \mathrm{DC}$ grid are also included in the model and show an important effect on the OPF solution. The case studies are conducted in a 5-terminal AC/DC meshed grid and point out that the CFC has a reduced capability to reduce the operating costs of the system if there are no overloads in the DC lines. However, when the power limit of one of the lines is reached, the CFC can redirect part of the DC current and, therefore, reduce the operating costs compared to the case without CFC.

Keywords: Optimal Power Flow, Current Flow Controller, meshed AC/DC grid, Cost minimization.
\end{abstract}

Email address: joan.sau@citcea.upc.edu . Tel. +34 934016724 Fax. +34 934017433 (Joan Sau-Bassols) 


\section{Nomenclature}

Upper-case letters are used for denoting parameters and sets. Lower-case letters denote variables and indexes. The superscripts ${ }^{a c}$ and ${ }^{d c}$ are used to denote $\mathrm{AC}$ and DC variables, respectively. The letters in bold indicate vectors or matrices.

Indexes, sets and subsets

$(m, n, o) \in \mathcal{C} \quad$ CFC buses

$(f, h, i, j, k) \quad$ buses, running from 1 to $B$

$g \in \mathcal{G} \quad$ generating units, running from 1 to $G$

$v \in \mathcal{V} \quad$ VSC converter, from 1 to $V$

$l \in \mathcal{L} \quad$ transmission branches, including lines and

transformers, running from 1 to $L$

$\mathcal{L}^{a c} \subset \mathcal{L} \quad$ subset of $\mathrm{AC}$ transmission lines

$\mathcal{L}^{d c} \subset \mathcal{L} \quad$ subset of DC transmission lines

$\mathcal{L}^{c f c} \subset \mathcal{L}_{d c} \quad$ subset of DC lines connected to the CFC

$\mathcal{L}^{n} \subset \mathcal{L}_{d c} \quad$ subset of DC lines not connected to the CFC

$\mathcal{B}^{a c} \subset \mathcal{B} \quad$ subset of $\mathrm{AC}$ buses

$\mathcal{B}^{d c} \subset \mathcal{B} \quad$ subset of DC buses

$\mathcal{B}^{c f c} \subset \mathcal{B}^{d c} \quad$ subset of DC buses with lines connected

to the $\mathrm{CFC}$

$\mathcal{B}^{n} \subset \mathcal{B}^{d c} \quad$ subset of DC buses whose lines are

not connected to the $\mathrm{CFC}$

$\mathcal{N}_{v}^{a c} \quad$ AC bus of converter $v$

$\mathcal{N}_{v}^{d c} \quad$ DC bus of converter $v$

$x \in \mathcal{X} \quad$ type of connection of the CFC: A, B or $\mathrm{C}$

Parameters 
$A_{g}, B_{g}, C_{g}$ cost coefficients of the generator

$N C_{p}, N C_{q}$ costs of non-served active and reactive power

$R_{l} \quad$ resistance of line $l$

$G_{l} \quad$ conductance of line $l$

$B_{l} \quad$ susceptance of line $l$

$B_{l}^{s h t} \quad$ half total line charging susceptance of line $l$

$G_{i j}^{a c} \quad$ AC network conductance matrix

$G_{i j}^{d c} \quad$ DC network conductance matrix

$B_{i j}^{a c} \quad$ AC network susceptance matrix

$A_{v}, B_{v} \quad$ independent and linear terms of the losses of converter $v$

$C_{v}^{r e c}, C_{v}^{i n v}$ quadratic terms of the losses of converter $v$ acting as rectifier/inverter

$\bar{S}_{l}, \bar{P}_{l} \quad$ flow limits on transmission line $l$

$\bar{I}_{v} \quad$ maximum current allowed through the converter $v$

$\bar{P}_{g}, \underline{P}_{g} \quad$ active power limits of the generator $g$

$\bar{Q}_{g}, \underline{Q}_{g} \quad$ reactive power limits of the generator $g$

$\bar{V}_{i}, \underline{V}_{i} \quad$ voltage limits at bus $i$

$\bar{E}, \underline{E} \quad$ voltage limits of the $\mathrm{CFC}$

$\bar{D}, \underline{D} \quad$ duty cycle limits of the switch $\mathrm{S} 1$ and $\mathrm{S} 3$ of the CFC

$P_{D i}, Q_{D i} \quad$ active and reactive power demand at bus $i$

\section{Variables}

$p_{l}, \rho_{v} \quad$ active power of line $l$, power losses of converter $v$

$p_{i}, q_{i} \quad$ active and reactive power injected at bus $i$

$n p_{i}, n q_{i}$ non-served active and reactive power at bus $i$ 
$v_{i}^{a c}, \theta_{i} \quad \mathrm{AC}$ voltage magnitude and phase angle at bus $i$

$v_{i}^{d c} \quad$ DC voltage magnitude at bus $i$

$p_{g}, q_{g} \quad$ active and reactive power generation of generator $g$

$i_{v}^{i n v}, i_{v}^{r e c}$ module of phase current of converter $v$ operating

as an inverter and rectifier respectively

$i_{i j}^{d c} \quad$ DC current circulating from bus $i$ to $j$

$v_{i j}^{d c} \quad$ Average DC voltage applied by the CFC between

bus $i$ and $j$

$e_{x} \quad$ mean voltage of the capacitor of the CFC

connected as $x$

$d_{x} \quad$ duty cycle of the switch S1 or S3 of the CFC

connected as $x$

\section{Introduction}

The increasing worldwide installation of Voltage Source Converter (VSC) based High Voltage Direct Current (HVDC) systems can be understood due to the advantages that this technology offers for transmitting large amounts of power over long distances or through undersea/underground cables [1]. The benefits of VSC-HVDC transmission systems lie in the lower footprint, black-start capability and decoupled control of active and reactive power [2]. Nowadays, those links are built as point-to-point connections using Modular Multilevel Converters (MMC) [3]. With the addition of more terminals or the interconnection of the existing links, the system can evolve into a Multi-Terminal (MT) HVDC system, bringing more flexibility, a concept that is being considered in Europe but is a reality in China, where two MT-HVDC grids are in operation [4]. If more lines are added into the MT system it may eventually become a meshed HVDC grid, which can provide additional flexibility and redundancy to the transmission system [5]. The European Supergrid $[6,7]$ falls into this concept, and it is being thought 
as the interconnection by means of an HVDC grid of the different European states, allowing the integration of various renewable resources. The previous concept provides a number of advantages compared to point-to-point HVDC and HVAC, but requires standardisation and coordination [1]. Currently, the first meshed HVDC grid worldwide is being designed in China, the so-called Zang-Bei project, which is meant to provide renewable power supply to Beijing. In the first phase, 4 terminals in a ring connection are planned, with 2 more coming in the second phase [8].

Those meshed HVDC grids may be embedded in the conventional AC grid, alleviating the power transmission and forming hybrid AC/DC grids. If HVDC grids become more common and the number of HVDC interconnections increase, this may complicate the power flow control within the DC grid and its operation can be restricted in case of bottlenecks or overloads as it happens in AC grids. These situations can be mitigated if additional devices known as Flexible DC Transmission Systems (FDCTS) [9], the equivalent of Flexible AC Transmission Systems (FACTS) for DC grids, are introduced. The FDCTS are expected to be installed in the critical points of the network, where the overloads may restrict the overall operation of the system, the same that happens with FACTS in the AC grid. Among the FDCTS, the Power Flow Controllers (PFC) or Current Flow Controllers (CFC) can be used to regulate the current circulating through the DC transmission lines and alleviate the congestions of the HVDC grid $[10,11]$.

CFCs are thought as power electronics converters of reduced size compared with the VSC terminals that allow to control the DC current through the transmission lines [12]. They are connected in series and their operation principle is based on applying variable voltages in series with the lines. The number of proposed CFCs in the literature is increasing and the diverse topologies can be gathered in variable resistances [11], DC/AC converters $[12,13]$ and DC/DC converters [14]-[22]. Among them, DC/DC based CFCs present some advantages since they are connected between DC lines within the grid and they do not require insulation transformers to be connected to the AC grid. DC/DC CFCs, also known as interline CFCs, are the group with more proposals in the 
literature, ranging from devices able to operate with bidirectional currents $[14,15,16$, 17, 18] to simpler topologies with unidirectional capability [19]. All the concepts are based on exchanging power between DC lines as a means to generate variable voltage sources that can affect the current distribution within the DC grid. The unidirectional topologies are based on the premise that not all the current flows circulating though the lines of the DC grid are going to be reversed. Due to this, distributed CFC approaches with a number of devices strategically installed in the DC grid may be more convenient with simpler CFC structures, with less switches and lower cost [20]. Besides, when considering larger DC grids with many nodes and interconnections, multi-port CFC structures which can be connected to multiple lines are also being proposed [21, 22].

As CFC devices introduce additional degrees of freedom into the DC grids, their role must be considered in Optimal Power Flow (OPF) problems in the AC/DC grids where they are installed. The problem of finding the Power Flow (PF) solution for the case of hybrid network with VSC-MTDC systems is relatively new [23, 24]. In addition to the PF problem, the Optimal Power Flow (OPF) was firstly introduced by Carpentier in 1962 [25]. The goal is to find the optimal scheduling of the generators while taking into account network constraints (capacity limits and security constraints) [26]. In order to assess and fully exploit the potential impact of VSC-MTDC, traditional models that were developed for AC systems need to be extended to cope with hybrid AC/DC networks. The impact on power flows derived from point-to-point interconnections between HVAC and HVDC systems is even more notable in case of having a meshed MTDC system overlaying an AC grid, and the control strategies of the converters become crucial. VSC allows flexible and independent active and reactive power control [6], and its forced-commutated feature makes it ideal to connect wind farms at distance as it can mitigate the propagation of voltage and frequency deviations caused by wind variations. However, the operating principles of VSC are completely different from those of Current Source Converter (CSC). Therefore, new algorithms need to be developed for VSC-HVDC control and power flow studies. Several research works have been proposed to include VSC-MTDC systems into power flow calculation of an hy- 
brid AC/DC system as previously mentioned. However, fewer studies take into account VSC-MTDC systems under an optimization context: [23] applies second-order cone programming (SOCP) technique, [27] solves the hybrid network using the Primal-Dual Interior Point (PDIP) algorithm, with predefined control strategies for DC networks, as well as modified Jacobian and Hessian matrices, and [34] utilizes Interior Point Optimizer (IPOPT) to seek solutions for the non-linear model built in General Algebraic Modelling System (GAMS). [28] has proposed an extended OPF model to consider loss modelling of different converter operation modes (inverter or rectifier).

To the best knowledge of the authors, the CFCs have only been included in an OPF problem in [29] and [30]. The PFC considered in [29] for the study is a AC/DC converter, which is used to reduce the curtailment of wind power in a DC grid interconnecting wind power plants. In [30], different types of CFCs are analysed and the optimum location to install the devices and ensure static security is assessed.

The present paper analyses the impact of an interline DC/DC based CFC on the OPF solution in an hybrid AC/DC meshed grid in steady-state. The purpose of the paper is to analyse if the $\mathrm{CFC}$ is able to reduce the operating costs of the hybrid $\mathrm{AC} / \mathrm{DC}$ meshed grid by alleviating the congestions in the DC lines. The work considers the unidirectional CFC topology introduced in [19] and quantifies the savings that the $\mathrm{CFC}$ can bring regarding the operating generator costs of the $\mathrm{AC} / \mathrm{DC}$ grid with and without overloaded lines. The previous CFC structure has a simple structure specially convenient for distributed CFC approaches with less switches than a bidirectional CFC [20]. This work does not study the best location of the CFC within the grid, instead it considers the installation of the device into one of the DC nodes and focuses the analysis in the connection arrangement of the CFC to the grid, due to its current unidirectionality. The modelling of the CFC is explained, detailing the corresponding constraints of the aforementioned topology and also its physical limits. The dynamic validation of the CFC topology under study is not included since it is already provided in [15]. The modelling of the hybrid AC/DC grid follows the same approach as in [28], also including the losses of the VSCs into the OPF problem. Two case studies 
are considered in order to evaluate the impact of the CFC in the hybrid AC/DC grid: Case study 1 analyses different demand levels that do not overload the DC lines and evaluates if the $\mathrm{CFC}$ can contribute to the $\mathrm{OPF}$ problem even if it is not required to alleviate any congestion. Case study 2 considers demand levels that imply DC line overloads and investigates the effect of the CFC when alleviating those congestions.

\section{Current Flow Controller}

\subsection{Series interline $D C / D C$ based $C F C$}

This section presents the CFC considered for this study, which is the interline DC/DC based CFC introduced in [19]. The scheme of the aforementioned device is shown in Fig. 1.

[Figure 1 about here.]

It can be seen that the device consists on two switches composed of a diode in series with an Insulated Gate Bipolar Transistor (IGBT) in order to obtain a reverse blocking voltage device. It also includes a capacitor that can have positive or negative voltage polarity. Two different topologies are shown in Fig. 1 depending whether two currents are entering the device (see Fig. 1(a)) or two currents are going out from the converter (see Fig. 1(b)). The operation of the converter consists on exchanging power between the two lines where it is connected, and by doing so, it applies variable voltage sources on the lines that allow to regulate the DC currents.

\section{2. $C F C$ losses}

The CFCs are thought as small converters, if compared with the VSC stations, whose rating is less than $10 \%$ of a VSC station [12]. Therefore, the losses of the CFC device are expected to be lower than VSC losses. From [14], it can be expected that the power losses of those devices will be in order of tens of $\mathrm{kW}$. The losses of a DC/DC interline $\mathrm{CFC}$ are calculated in [20], reporting values up to $23 \mathrm{~kW}$, which represent the

$0.002 \%$ of the rating of a VSC station. Consequently, this work considers the losses of the $\mathrm{CFC}$ to be negligible for the OPF problem. 


\subsection{CFC modelling}

In order to integrate the CFC into the OPF model, its average model, derived in [19], is used. This model represents the two CFC structures shown in Fig. 1 and is illustrated in Fig. 2. The circuit comprises two voltage sources that the CFC is applying in series on the lines, whose value depends on the duty cycle of the switches, $d_{x}^{d c}$, and the average voltage of the capacitor, $e_{x}^{d c}$ :

$$
v_{n m}^{d c}=\left(1-d_{x}^{d c}\right) e_{x}^{d c} \quad v_{o m}^{d c}=-d_{x}^{d c} e_{x}^{d c}
$$

[Figure 2 about here.]

The subscript $X$ defines the way the CFC is connected to the DC grid, which is explained in Section 4.2.1. Also, in order to keep the balance of its capacitor, the following equation must be met for the CFC structure of Fig. 1(a):

$$
-d_{x}^{d c} i_{o m}^{d c}+\left(1-d_{x}^{d c}\right) i_{n m}=0
$$

and for the CFC structure in Fig. 1(b):

$$
-d_{x}^{d c} i_{m o}^{d c}+\left(1-d_{x}^{d c}\right) i_{m n}=0
$$

Then, the duty cycle can be expressed as a relation of the currents through the CFC, for both CFC structures, which are illustrated in (4) and (5), respectively:

$$
\begin{aligned}
& d_{x}^{d c}=\frac{i_{n m}^{d c}}{i_{n m}^{d c}+i_{o m}^{d c}} \\
& d_{x}^{d c}=\frac{i_{m n}^{d c}}{i_{m n}^{d c}+i_{m o}^{d c}}
\end{aligned}
$$

This implies that setting a duty cycle for the switches defines a certain relation between the lines currents in steady-state. The CFC has one degree of freedom, so that, whenever the duty cycle (current relation) is defined, the capacitor voltage will adapt its value to provide such a change in the DC grid, assuming that the device limits are not exceeded [19]. Note that, depending on how the CFC is connected to the DC grid, the current relation that the duty cycle brings will affect different line currents. 


\subsection{Operation limits}

\subsubsection{Topology constraints regarding line currents}

Due to the inherent structure of the CFC converter, which is depicted in Fig. 1, while operating with the structure of Fig. 1(a) or 1(b), the current direction must be the one shown by the corresponding arrow and cannot be reversed. This fact introduces several constraints when implementing the CFC model in the OPF as with a defined CFC structure in a certain location, the DC currents of the lines where it is connected must have certain directions. Therefore, considering the CFC structure of Fig. 1(a):

$$
\begin{aligned}
& i_{n m}^{d c} \geq 0 \quad \forall x \in \mathcal{X} \\
& i_{o m}^{d c} \geq 0 \quad \forall x \in \mathcal{X}
\end{aligned}
$$

Regarding the CFC in Fig. 1(b):

$$
\begin{array}{ll}
i_{m n}^{d c} \geq 0 & \forall x \in \mathcal{X} \\
i_{m o}^{d c} \geq 0 & \forall x \in \mathcal{X}
\end{array}
$$

\subsubsection{Voltage limit of the $C F C$}

CFCs are devices based on power electronics rated for a certain voltage. The voltage level that the switches must withstand are defined by the capacitor voltage of the CFC [19]. Due to this reason, the following restriction is required:

$$
\underline{E} \leq e_{x} \leq \bar{E} \quad \forall x \in \mathcal{X}
$$

\subsubsection{Duty cycle limits of the CFC}

According to the operation of the CFC topology considered, the duty cycle of the switches that is used in the average model is comprised between 0 and 1 :

$$
\underline{D} \leq d_{x} \leq \bar{D} \quad \forall x \in \mathcal{X}
$$




\section{Voltage Source Converters (VSC)}

VSC stations are the elements used to interconnect the DC and AC networks and the structures based on MMCs are likely to be the key technology for VSC-HVDC transmission [1]. MMCs are based on standard modular structures (submodules), whose number can be increased in order to achieve higher voltages [3]. Besides, the submodules allow to modulate $\mathrm{AC}$ waveforms which are really close to a sinusoidal waveform, therefore, reducing the AC filter requirements. Also, its relatively low switching frequency permits a reduction in the switching losses [31]. The VSC stations also incorporate reactors to connect the VSC to the AC grid, harmonic filters and an AC transformer [32]. For simplicity, all variables presented in this section are in [p.u.], and the units of the corresponding parameters are adapted accordingly.

\subsection{VSC equivalent model}

The typical approach to represent the VSC is to use a controllable voltage source, which in phasor notation is described as $\underline{v}_{c}=v_{c} \angle \theta_{c}[33,34]$. The model is illustrated in Fig. 3, where the voltage source is connected by means of a phase reactor $\left(\underline{z}_{c}=r_{c}+j x_{c}\right)$ to a lossless shunt filter $\left(\underline{z}_{f}=-j x_{f}\right)$. The voltage at this node is defined as $\underline{v}_{f}=v_{f} \angle \theta_{f}$ and the transformer, which connects this node to the AC network, is modelled as a series impedance $\left(\underline{z}_{t f}=r_{t f}+j x_{t f}\right)$. The voltage of the AC network in the Point of Common Coupling (PCC) is $\underline{v}_{s}=v_{s} \angle \theta_{s}$ and its magnitude and angle are equivalent to the voltage and angle in the corresponding $\mathrm{AC}$ bus $i, v_{i}^{a c}$ and $\theta_{i}$, respectively. The apparent power extracted from the DC grid is $\underline{s}_{c}=p_{c}+j q_{c}$ and the apparent power after the filter and the transformer, which is injected into the AC network, is $\underline{s}_{s}=p_{s}+j q_{s}$.

[Figure 3 about here.]

The power balance in the converter is established according the following equation:

$$
p_{v}^{d c}+p_{v}^{a c}+\rho_{v}=0, \quad \forall v \in \mathcal{V}
$$

where, $p_{v}^{a c}$ corresponds to $p_{c}$, the real part of the apparent power at the bus c. Both $p_{v}^{d c}$ and $p_{v}^{a c}$ are assumed to be positive when the power is injected in the DC or AC grid, respectively, and the power losses $\rho_{v}$ only take positive values. 


\subsection{VSC losses modelling}

In the VSC stations, a percentage of the transmitted power is lost due to the converter losses. Those losses in a MMC come from the semiconductor losses in each of the SMs, arm reactors, phase reactors and transformers. Several works in the literature provide different methods for calculating the power losses in a MMC [35, 36, 37, 38]. However, such complex formulations cannot be included easily in an OPF model. Due to this, it is a common practice to use a polynomial expression to model the converter losses [33]. In [28], the effect of considering different models to represent the VSC losses is investigated. The conclusion obtained in [28] is that the OPF solution highly depends on the losses model employed. Therefore, this work considers an accurate way to represent the converter losses, that uses a quadratic function, which depends on the direction and value of the phase current circulating through the converter.

$$
\rho_{v}=A_{v}+B_{v}\left|i_{v}\right|+C_{v}^{i n v}\left(i_{v}^{i n v}\right)^{2}+C_{v}^{r e c}\left(i_{v}^{r e c}\right)^{2}
$$

where, $i_{v}=i_{v}^{i n v}-i_{v}^{r e c}$ and $i_{v}^{i n v}, i_{v}^{r e c} \geq 0 ; A_{v}, B_{v}$ and $C_{v}^{r e c} C_{v}^{i n v}$ are the corresponding loss coefficients.

\subsection{VSC operational limits}

The operation of the VSCs presents several limits, which are described in [27] in order to ensure the proper operation of the converter. The limits included in this study are explained below.

\subsubsection{Current limit of the converter}

This limit aims to avoid damages in the switches of the VSC. In normal operation, the current through the IGBTs depends on the phase current of the VSC. The IGBTs are rated for a certain amount of current, so a maximum and a minimum value for the current that the VSC can withstand is introduced:

$$
-\bar{I}_{v} \leq i_{v} \leq \bar{I}_{v}, \quad \forall v \in \mathcal{V}
$$




\subsubsection{Relation between $D C$ and $A C$ voltage}

Concerning the operation of a VSC, there is a limit on the maximum AC voltage that can be modulated for a given DC voltage [39]. To introduce this constraint, the $\mathrm{AC}$ voltage and the $\mathrm{DC}$ voltage are related with a value $k_{v}$ as shown in (15). The $k_{v}$ value is set to 1.1 as in [34], though, it can be modified according to the modulation method employed.

$$
v_{c} \leq k_{v} v_{i}^{d c} \quad \forall v \in \mathcal{V}, c \in \mathcal{N}_{v}^{a c}, i \in \mathcal{N}_{v}^{d c}
$$

By setting this limit and a limit on the $\mathrm{DC}$ voltage value, the $\mathrm{AC}$ maximum voltage is imposed.

3.3.3. Boundaries of active and reactive power injected to the AC network

$$
p_{v}^{2}+q_{v}^{2}=\left(v_{c} i_{v}\right)^{2} \quad \forall c \in \mathcal{N}_{v}^{a c}, v \in \mathcal{V}
$$

\section{Optimal power flow modelling of an hybrid AC/DC network}

This section presents the complete mathematical formulations for the proposed nonlinear programming OPF model. For simplicity, all variables presented in this section are in [p.u.], and the units of the corresponding parameters are adapted accordingly.

\subsection{AC Network Constraints}

\subsubsection{AC Power Flow Equations}

Every bus $i$ of the AC grid is defined by its voltage magnitude $v_{i}^{a c}$ and phase angle $\theta_{i}$. By denoting $\theta_{i j}=\theta_{i}-\theta_{j}$, power injections at node $i$ and power flows (from $i$ to $j$ ) on branch $l$, i.e., $(i, j) \in \mathcal{L}^{a c}$, are $[25]$ :

$$
\begin{gathered}
p_{i}^{a c}=v_{i}^{a c} \sum_{j \in \mathcal{B}^{a c}} v_{j}^{a c}\left[G_{i j}^{a c} \cos \left(\theta_{i j}\right)+B_{i j} \sin \left(\theta_{i j}\right)\right], \forall i \in \mathcal{B}^{a c} \\
q_{i}^{a c}=v_{i}^{a c} \sum_{j \in \mathcal{B}^{a c}} v_{j}^{a c}\left[G_{i j}^{a c} \sin \left(\theta_{i j}\right)-B_{i j} \cos \left(\theta_{i j}\right)\right], \forall i \in \mathcal{B}^{a c} \\
p_{l}^{a c}=\left(v_{i}^{a c}\right)^{2} G_{l}-v_{i}^{a c} v_{j}^{a c}\left[G_{l} \cos \left(\theta_{i j}\right)+B_{l} \sin \left(\theta_{i j}\right)\right]
\end{gathered}
$$




$$
q_{l}^{a c}=-\left(v_{i}^{a c}\right)^{2}\left(B_{l}+B_{l}^{s h t}\right)-v_{i}^{a c} v_{j}^{a c}\left[G_{l} \sin \left(\theta_{i j}\right)-B_{l} \cos \left(\theta_{i j}\right)\right]
$$

Notice that transformers are modelled as pi sections with pre-defined tap ratios. Conservation of power are established through (21) and (22) taking into account active power injections from VSCs:

$$
\begin{aligned}
p_{i}^{a c} & =\sum_{g \in \mathcal{G}_{i}} p_{g}-P_{D i}+\sum_{v \in \mathcal{V}_{i}} p_{v}^{a c}+n p_{i}, \forall i \in \mathcal{B}^{a c} \\
q_{i}^{a c} & =\sum_{g \in \mathcal{G}_{i}} q_{g}-Q_{D i}+\sum_{v \in \mathcal{V}_{i}} q_{v}^{a c}+n q_{i}, \forall i \in \mathcal{B}^{a c}
\end{aligned}
$$

4.1.2. AC voltage limits

$$
\underline{V}_{i}^{a c} \leq v_{i}^{a c} \leq \bar{V}_{i}^{a c}, \quad \forall i \in \mathcal{B}^{a c}
$$

4.1.3. AC line capacity limit

$$
\left(p_{l}^{a c}\right)^{2}+\left(q_{l}^{a c}\right)^{2} \leq\left(\bar{S}_{l}^{a c}\right)^{2}, \quad \forall l \in \mathcal{L}^{a c}
$$

\subsection{DC network constraints}

\subsubsection{DC power flow equations}

$v_{i}^{d c}$ is the voltage of the bus $i$ of the DC grid. The line connecting a pair of DC buses is represented by its resistance $R_{l}=1 / G_{l}$. A symmetrical monopole configuration is assumed for the DC grid $(n=2)$.

Assuming that the CFC is located in node $i$ and it is applying voltages on the lines that interconnect node $i$ with nodes $j$ and $k$. Then, $i, j$ and $k \in \mathcal{B}^{c f c}$ and the rest of the buses $\in \mathcal{B}^{n}$. The DC lines connected directly to the CFC are $(i, j)$ and $(i, k)$ both $\in \mathcal{L}^{c f c}$ and the other lines $\in \mathcal{L}^{n}$. The real power injected at nodes $i, j$ and $k$ satisfy the following expressions, respectively:

$$
\begin{gathered}
p_{i}^{d c}=v_{i}^{d c} n \sum_{h \in \mathcal{B}^{n}} G_{i h}^{d c}\left(v_{i}^{d c}-v_{h}^{d c}\right)+p_{i j}^{d c}+p_{i k}^{d c} \\
p_{j}^{d c}=v_{j}^{d c} n \sum_{h \in \mathcal{B}^{n}} G_{j h}^{d c}\left(v_{j}^{d c}-v_{h}^{d c}\right)+p_{j i}^{d c}
\end{gathered}
$$




$$
p_{k}=v_{k}^{d c} n \sum_{h \in \mathcal{B}^{n}} G_{k h}^{d c}\left(v_{k}^{d c}-v_{h}^{d c}\right)+p_{k i}^{d c}
$$

where:

$$
\begin{aligned}
p_{i j}^{d c}= & \left(v_{i}^{d c}-v_{i j}^{c f c}\right) n G_{i j}\left(v_{i}^{d c}-v_{i j}^{c f c}-v_{j}^{d c}\right),(i, j) \in \mathcal{L}^{c f c} \\
& p_{j i}^{d c}=v_{j}^{d c} n G_{j i}\left(v_{j}^{d c}+v_{i j}^{c f c}-v_{i}^{d c}\right),(j, i) \in \mathcal{L}^{c f c} \\
p_{i k}^{d c}= & \left(v_{i}^{d c}-v_{i k}^{c f c}\right) n G_{i k}\left(v_{i}^{d c}-v_{i k}^{c f c}-v_{k}^{d c}\right),(i, k) \in \mathcal{L}^{c f c} \\
& p_{k i}^{d c}=v_{k}^{d c} n G_{k i}\left(v_{k}^{d c}+v_{i k}^{c f c}-v_{i}^{d c}\right),(k, i) \in \mathcal{L}^{c f c}
\end{aligned}
$$

Three connection arrangements are possible as depicted in Fig. 4 for the CFC located in bus $i$.

[Figure 4 about here.]

Depending on the type of connection, the average voltages applied by the CFC between bus $i$ and the lines that go to bus $j$ and $k$ are shown below. Considering connection CFC A (see Fig. 4(a)):

$$
v_{i j}^{c f c}=-\left(1-d_{A}^{d c}\right) e_{A}^{d c} \quad v_{i k}^{c f c}=d_{A}^{d c} e_{A}^{d c}
$$

Regarding connection CFC B, the voltages applied are:

$$
v_{i j}^{c f c}=-d_{B}^{d c} e_{B}^{d c} \quad v_{i k}^{c f c}=-e_{B}^{d c}
$$

And concerning connection $\mathrm{CFC} \mathrm{C}$, the expressions are:

$$
v_{i j}^{c f c}=e_{C}^{d c} \quad v_{i k}^{c f c}=\left(1-d_{C}^{d c}\right) e_{C}^{d c}
$$

Additionally, the equations that relate the DC currents with the duty cycle, (2) and (3), for each CFC must be included in the problem according to the CFC connection. Besides, the restrictions presented in Section 2.4 regarding CFC operation must be also taken into account.

The power of a generic bus $h$ not affected directly by the CFC is expressed as:

$$
p_{h}^{d c}=v_{h}^{d c} n \sum_{f \in \mathcal{B}^{n}} G_{h f}^{d c}\left(v_{h}^{d c}-v_{f}^{d c}\right), \forall h \in \mathcal{B}^{n}
$$


Also, the power through line $l$, which interconnects buses $(h, f)$ where the CFC is not connected, satisfies the following expression:

$$
p_{l}^{d c}=G_{h f} n\left[v_{h}^{d c}\left(v_{h}^{d c}-v_{f}^{d c}\right)\right], \quad \forall(h, f) \in \mathcal{L}^{n}
$$

Finally, the relation between the powers of the VSC and the power in each node is established according to:

$$
p_{i}^{d c}=p_{v}^{d c}, \quad \forall i \in \mathcal{B}^{d c}, \forall v \in \mathcal{V}
$$

\subsubsection{Bus Voltage Limits}

$$
\underline{V}_{i}^{d c} \leq v_{i}^{d c} \leq \bar{V}_{i}^{d c}, \quad \forall i \in \mathcal{B}^{d c}
$$

4.2.3. DC Transmission Line Capacity Limits

$$
-\bar{P}_{l}^{d c} \leq p_{l}^{d c} \leq \bar{P}_{l}^{d c}, \quad \forall l \in \mathcal{L}^{d c}
$$

\subsection{Additional VSC constraints}

As illustrated in Section 3.2, converter losses depend on whether it operates as a

rectifier or an inverter. As a result, in order to model such difference, the following equation is included to force converter phase current to have the same direction as the power injected to the corresponding AC bus:

$$
0 \leq I_{v} p_{v}^{a c}, \quad \forall v \in \mathcal{V}
$$

\subsection{Generator Capacity}

The limits of the generators are also included in the model, regarding active and reactive power:

$$
\begin{aligned}
& \underline{P}_{g} \leq p_{g} \leq \bar{P}_{g}, \quad \forall g \in \mathcal{G} \\
& \underline{Q}_{g} \leq q_{g} \leq \bar{Q}_{g}, \quad \forall g \in \mathcal{G}
\end{aligned}
$$




\subsection{Objective Function/Optimization Criterion}

The considered Objective Function (OF) minimizes the total operating costs plus the penalty from the non-served active and reactive power as shown in [28] assuming $n p_{i} \geq 0, n q_{i} \geq 0$. The cost coefficients of the generators are $A_{g}, B_{g}$ and $C_{g}$ and $N C_{p}$ and $N C_{q}$ represent the unitary costs of non-served real and reactive power, respectively. Other alternatives, such as minimization of network losses as in [27], could also be considered.

$$
\min \sum_{g \in \mathcal{G}}\left(C_{g}+A_{g} p_{g}+B_{g} p_{g}^{2}\right)+\sum_{i \in \mathcal{B}}\left(N C_{p} n p_{i}+N C_{q} n q_{i}\right)
$$

\section{Case studies}

This work considers two case studies to analyse the effect of the CFC when solving an $\mathrm{OPF}$ problem in a meshed $\mathrm{AC} / \mathrm{DC}$ grid. Both case studies consider the same AC/DC grid, which originates from [40], and an equal location of the CFC illustrated in Fig. 5. The AC/DC grid consists of $5 \mathrm{AC}$ buses and $3 \mathrm{DC}$ buses with the CFC located in DC bus 2. Each AC bus has its own active and reactive power demand and two generator units are located at bus 1 and 2. The parameters used for the case studies are the same as in [28], except for the ones detailed below.

Table 1 shows the parameters of the CFC used in the study. The nominal demand levels for each bus and case study are shown in Table 2 and the line impedances are extracted from [41] for AC and DC overhead lines.

[Figure 5 about here.]

[Table 1 about here.]

Regarding the CFC connection types described in Section 4.2.1, buses $i, j$ and $k$ are assigned to DC buses 2, 3 and 5, respectively in Fig. 5, and the CFCs are named according to the type of connection: A, B and C.

[Table 2 about here.] 


\subsection{Case study 1}

In Case study 1 the OPF problem is solved for different demand levels and considering the $\mathrm{AC} / \mathrm{DC}$ grid without $\mathrm{CFC}$ and with $\mathrm{CFC}$, where all the possible connections of the CFC presented in Section 4.2.1 are taken into account. For case study 1 both DC and $\mathrm{AC}$ cables are assumed to have a capacity limit of 800 MVA/MW. Table 3 depicts the results for Case study 1.

[Table 3 about here.]

The first column in Table 3 shows the value in [p.u.] of the demand in each bus (both for active and reactive power) according to the values of Table 2. The final power value used is the product of the [p.u.] of Table 3 and the nominal value in Table 2. Then, for each demand level, the OPF is solved considering that there is no CFC in the DC grid (No CFC); the CFC is installed in bus 2 and connected according option A ( $\mathrm{CFC} \mathrm{A}$ ), see Fig. 4; the $\mathrm{CFC}$ is connected according option $\mathrm{B}$ (CFC B); ad finally that the $\mathrm{CFC}$ is connected according option $\mathrm{C}(\mathrm{CFC} \mathrm{C})$. The value of the $\mathrm{OF}$ (operating costs plus the cost of non-served power) and the DC power circulating through lines 23 and 25 is presentedin the third and forth column, respectively. Finally, the average CFC voltage and the duty cycle applied to its switches are also shown, when the CFC is present, in the last two columns, respectively.

From Table 3, it can be seen that the OP value increases with the demand level of the buses, which is expected, since more power is flowing through the AC/DC grid. When comparing the OP value assuming no CFC in the grid and different connections of the CFC, the results are equivalent for al the power demands. The OF does not vary much comparing the cases without CFC and with CFC. For all the demand levels, the CFC A can provide the same OF as without CFC but not lower. On the other hand, the OF of CFCs B and C are slightly higher for all the demand levels, which means that the cost of operating the system is higher when considering the previous connections. The best options (lower OF) for each demand level are depicted in boldand correspond to having no $\mathrm{CFC}$ in the $\mathrm{DC}$ grid or the $\mathrm{CFC}$ with the connection type $\mathrm{A}(\mathrm{CFC} \mathrm{A})$ with equal $\mathrm{OP}$ value. 
It can be seen that CFC A applies no effect on the DC grid since the CFC voltage, $e_{x}$, is 0 and the powers through lines 23 and 25 are equivalent to the ones without CFC. In those situations, the CFC A is applying the Neutral Duty Cycle, which basically means that it is operating but no effect is applied on the line currents [19]. This fact points out, that the $\mathrm{OPF}$ is not able to find a operating point where the CFC can reduce the operating costs of the system.

When working with CFC B and C, the unidirectional restrictions of the CFC topology regarding the directions of $\mathrm{DC}$ currents imply that both $\mathrm{CFC}$ must reduce the current of one of the DC lines to 0 in order to operate. Therefore, CFC B and C need to apply a certain CFC voltage as it can be seen in column $e_{x}$ and the duty cycle is either 0 or 1 . Due to this change in the DC line currents, the losses of the system increase as reported in [15], which results in higher values of the OF. Another outcome is that when increasing the demand of the buses, more power is circulating within the system and therefore, higher CFC voltages are required to redirect the DC currents.

For the previous results, the non-served power is 0 for all the buses, which means that the system is able to deliver the demanded power.

\subsection{Case study 2}

In Case study 2 the OPF problem is solved also for different demand levels and considering the $\mathrm{AC} / \mathrm{DC}$ grid without $\mathrm{CFC}$ and with $\mathrm{CFC}$, where all the possible connections of the CFC presented in Section 4.2.1 are also taken into account. However, for this Case study 2, the DC and AC cables are assumed to have a power limit of 800 MVA, except DC line 25, whose power limit is set at $200 \mathrm{MW}$ in order to investigate the capabilities of the CFC when power limit restrictions take place. The power demand is higher in this case study if compared with the one in the previous section, in order to illustrate as well the effect of having power non-served in some buses.

Table 4 shows the results of Case study 2, illustrating the same variables as in Case study 1. Additionally, the last column shows the non-served power in bus $5, n p_{5}$, which is an important factor affecting the OF. In the rest of the buses, the non-served power is zero, which means that all power demands are satisfied. The results are depicted in 
a scale of gray ordered from the lowest OP to the highest in the following manner for each demand level: bold black, black, dark gray and light gray.Therefore, the results in bold black show the CFC option that provides lower operation costs of the system and higher savings.

[Table 4 about here.]

The OP values for this case study are higher compared to the Case study 1 due to the higher demand level of some buses, leading to more power circulating through the system.

For all demand levels, the approach that can provide a higher reduction in the operating costs (OP) is the CFC A. The maximum value of DC power circulating through line 25 is reached (200 MW) except for CFC B, where the current through line 25 must be reduced to 0 in order to operate.

For demand level 1.00, CFC A can provide a slight reduction in the OF compared to the cases without CFC. Regarding CFC B and C, their OF is higher since they must reduce the current through one of the lines to 0 to be able to operate due to the unidirectional current restriction of the device. Therefore, their duty cycle is either 0 or 1 depending. Considering CFC B, the power cannot circulate through line 25, and the same happens for $\mathrm{CFC} \mathrm{C}$ for line 23, where the power flow is 0 . The situation is more critical for CFC C, since the other DC to transmit the power is limited to 200 MW. This fact leads to high operation costs as the power must circulate through a non-optimum path.

Looking at demand level 1.01, both CFC A and B can provide a OF reduction compared to the case without CFC. It is important to notice that the best approach among the two connection arrangements is CFC A with a lower OP. Also, for CFC A, the voltage that the CFC must apply, $e_{x}$, is much lower than the one of CFC B. A higher CFC voltage increases the ratings of the switches of the device, leading to a converter more costly. Regarding CFC C, it is not a reasonable option for this specific case study because it must reduce the current of line 23 to 0 to be able to operate, and at the same time the power through line 25 cannot be higher than $200 \mathrm{MW}$. This fact 
leads to a low efficiency configuration where 9.6 MW cannot be served to bus 5 . The non-served power to bus 5 has a strong penalty in the OP as it can be seen for CFC C, where the OP is much higher than the other options.

Considering higher demand levels (from 1.02 to 1.05), the cases without CFC and CFC $\mathrm{C}$ have some non-served power in node 5 that increases considerably the value of the $\mathrm{OF}$. However, among the $\mathrm{CFC} \mathrm{A}$ and $\mathrm{B}, \mathrm{CFC} \mathrm{A}$ can bring a slight reduction in the operating costs, since it changes less the current flows within the DC grid. Also, the voltage that CFC B has to apply is significantly higher than CFC A.

The power flows of the system considering Case study 2 and demand level 1.03 are illustrated in Fig. 6. The different colours show the active power that circulates through each line considering No CFC, CFC A and CFC B. CFC C is not included in Fig. 6 because as shown in Table 4 it is not a reasonable option to reduce the operating costs.

Regarding demand level 1.05, either with or without CFC, the system is not able to deliver all the power to bus 5 , though the non-served power is much lower with CFC $\mathrm{A}$ and $\mathrm{B}$ compared to the cases without $\mathrm{CFC}$ or with $\mathrm{CFC} \mathrm{C}$. For this scenario, the savings than the CFC A and B can bring to the system are in the order of $24 \%$ of the costs compared to the case without CFC.

[Figure 6 about here.]

\subsection{Discussion of the case studies}

The previous case studies showed the capability of the CFC to reduce the operating costs of an meshed AC/DC grid as a function of the connection arrangement of the device under different demand levels. The results are significantly different if restrictions on the power limit of the lines take place. For the first case study, where the current of the lines do not exceed the limits, the CFC cannot reduce the operating costs compared to the case without CFC. However, when a restriction in one of the DC lines takes place, the $\mathrm{CFC}$, specifically the connection arrangement A (CFC A), presents a considerable capability to reduce the operating costs. It redirects part of the DC current through

other lines and maintains the current circulating through the overloaded line to its 
allowable value. Also, the non-served power that introduces a penalty in the OF, plays and important role when assessing the capability of the CFC.

The previous results have been obtained in a system with $5 \mathrm{AC}$ and $3 \mathrm{DC}$ buses forming a meshed $\mathrm{AC} / \mathrm{DC}$ grid in order to analyse in detail the different aspects. Nevertheless, a system with more nodes can also be employed using the same methodology applied in this work and the operating costs reduction when using CFCs and their connection arrangement can be assessed. Also, in a larger system, other concerns may arise, for instance, more than one CFC may be installed in the system. Then, the coordination among the different CFCs becomes also a concern and the unidirectional CFC topology can be a convenient option due to its simple structure. In such a situation, the optimum location of the different CFC devices plays an important role, as well.

\section{Conclusion}

This paper has presented the insertion of an interline CFC in an OPF problem of a meshed hybrid AC/DC grid. The impact of the CFC in the OPF solution has been analysed showing that in certain situations the CFC can reduce the operating costs of the system. The modelling of the CFC in the OPF is explained along with the modelling of the VSCs and the mathematical formulation of the $\mathrm{AC} / \mathrm{DC}$ grid $\mathrm{OPF}$ problem. Two case studies have been conducted to investigate the impact of the CFC in the OPF solution taking into account different demand levels in the buses and connection arrangements of the $\mathrm{CFC}$. When the power limit of the lines is not reached, the CFC has no capability to reduce the operating costs since changing the grid currents increase the losses of the system, and therefore, the costs. However, when there is an overload in one of the lines, the CFC can be used to redirect part of the power to a less congested line and keeping the power through the congested line at an acceptable value, providing then, a reduction in the operating costs. The non-served power has also a considerable effect on the OPF solution. For the considered CFC topology, the importance of the connection of the CFC to the DC grid is of great importance as different connections lead to different results in the operating costs and to different voltage ratings of the CFC. 


\section{Acknowledgements}

This work was supported by the Spanish Ministry of Science, Innovation and Universities under the Projects ENE2015-67048-C4-1-R and RTI2018-095429-B-I00. This work was co-financed by the European Regional Development Fund.

[1] D. Van Hertem, O. Gomis-Bellmunt and J. Liang, HVDC Grids: For Offshore and Supergrid of the Future, IEEE Press Series on Power Engineering, Ed. John Wiley \& Sons, 2016.

[2] D. Jovcic and K. Ahmed, High Voltage Direct Current Transmission: Converters, Systems and DC Grids, University of Aberdeen, UK, Wiley 2015.

[3] A. Lesnicar and R. Marquardt, An Innovative Modular Multilevel Converter Topology Suitable for a Wide Power Range, in IEEE PowerTech Conference, Bologna 2003, pp. 1-6.

[4] G. Buigues, V. Valverde, A. Etxegarai, P. Eguia, and E. Torres, "Present and future multiterminal HVDC systems : current status and forthcoming developments," in International Conference on Renewable Energies and Power Quality (ICREPQ17), vol. 1, no. 15, pp.83-88, 2017.

[5] D. Van Hertem and M. Ghandhari, "Multi-terminal VSC HVDC for the European supergrid: Obstacles", Renewable and Sustainable Energy Reviews, vol. 14, issue 9, pp. 3156-3163, Dec. 2010.

[6] N. Flourentzou, V. Agelidis, G. Demetriades, VSC-based HVDC powertransmission systems: an overview, IEEE Trans. Power Electron. vol. 24 no. 3 pp. 592602, Mar. 2009.

[7] Friends of the Supergrid, "Roadmap to the Supergrid Technologies," Tech. Rep., 2016. [Online]. Available: https: //www.friendsofthesupergrid.eu/media/technology/. 
[8] ABB, "Zhangbei: The world's first DC-grid with HVDC Light." [Online]. Available: https://new.abb.com/systems/hvdc/references/ zhangbei.

[9] K. Rouzbehi, A. Miranian, A. Luna and P. Rodriguez, "Towards fully controllable multi-terminal DC grids using flexible DC transmission systems," in Conversion Congress and Expo. (ECCE), 2014, pp. 1-5.

[10] E. Veilleux and B. Ooi, "Power flow analysis in multi-terminal HVDC grid," in Power Systems Conf. and Expo. (PSCE), 2011, pp. 1-7.

[11] Q. Mu, J. Liang, Y. Li and X. Zhou, "Power flow control devices in DC grids," in Power and Energy Society General Meeting IEEE, 2012, pp. 1-7.

[12] E. Veilleux and B. Ooi. "Multi-terminal HVDC with thyristor power-flow controller," IEEE Trans. Power Del., vol. 27, no. 3, pp. 1205-1212, Jul. 2012.

[13] S. Balasubramaniam, J. Liang and C.E. Ugalde-Loo, "An IGBT based series power flow controller for multi-terminal HVDC transmission," in 49th Int UPEC, ClujNapoca, 2014, pp. 1-6.

[14] C. Barker and R. Whitehouse, "A current flow controller for use in HVDC grids," in IET Int. Conf. on $A C$ and DC Power Trans. (ACDC), 2012, pp. 1-5.

[15] J. Sau-Bassols, E. Prieto-Araujo and O. Gomis-Bellmunt, "Modelling and control of an interline Current Flow Controller for meshed HVDC grids," IEEE Trans. Power Del. vol. 32, issue 1, pp. 11-22, Feb. 2017.

[16] F. Hassan, R. King, R. Whitehouse and C. Barker, "Double modulation control (DMC) for dual H-bridge current flow controller (2B-CFC)," in 17th European Conf. on Power Electron. and Applications (EPE'15 ECCE-Europe), Geneva 2015, pp. 19 .

[17] S. Balasubramaniam, C. Ugalde-Loo, J. Liang, T. Joseph, R. King and A. Adamczyk, "Experimental Validation of Dual H-Bridge Current Flow Controllers for 
Meshed HVdc Grids," IEEE Trans. Power Del. vol. 33, issue 1, pp. 381-392, Sep. 2017.

[18] W. Chen, X. Zhu, L. Yao, G. Ning, Y. Li, Z. Wang, W. Gu and X. Qu, "A Novel Interline DC Power-Flow Controller (IDCPFC) for Meshed HVDC Grids," IEEE Trans. Power Del. vol. 31, issue 4, pp. 1719-1727, Aug. 2016.

[19] J. Sau-Bassols, E. Prieto-Araujo, O. Gomis-Bellmunt and F. Hassan, "Series interline DC/DC Current Flow Controller for meshed HVDC grids," IEEE Trans. Power Del. vol. 33, issue 2, pp. 881-891, Apr 2017.

[20] J. Sau-Bassols, E. Prieto-Araujo, O. Gomis-Bellmunt and F. Hassan, "Selective Operation of Distributed Current Flow Controller Devices for Meshed HVDC Grids", IEEE Trans. Power Del., Early access, 2018.

[21] H. Y. Diab, M. I. Marei and S. B. Tennakoon, "Operation and control of an insulated gate bipolar transistor-based current controlling device for power flow applications in multiterminal high-voltage direct current grids," IET Power Electron. vol. 9, issue 2, pp. 305-315, Feb. 2016.

[22] M. Ranjram and P. W. Lehn, "A Multiport Power-Flow Controller for DC Transmission Grids," IEEE Trans. Power Del. vol. 31, issue 1, pp. 389-396, Feb. 2016.

[23] M. Baradar, M. Hesamzadeh, M. Ghandhari, "Second-order cone programming for optimal power flow in vsc-type ac-dc grids", IEEE Trans. Power Syst. vol. 28, issue 4,pp. 42824291, Nov. 2013.

[24] M. H. Okba, M. H. Saied, M. Z. Mostafa, and T. M. Abdel-Moneim, "High voltage direct current transmission - A review", part I, in 2012 IEEE Energytech, pp. 17, May 2012.

[25] A.J. Wood, B.F. Wollenberg, Power Generation, Operation, and Control, 2nd ed., John Wiley \& Sons, Nov. 2012. 
[26] M. Aragüés-Peñalba, A. Egea-Àlvarez, S. Galceran-Arellano and O. GomisBellmunt, "Optimal power flow tool for mixed high-voltage alternating current and high-voltage direct current systems for grid integration of large wind power plants", IET Renewable Power Generation, vol. 9, issue 8, pp. 876-881, Nov. 2015.

[27] J. Cao, W. Du, H. Wang, S. Bu, "Minimization of transmission loss in meshed AC/DC grids with VSC-MTDC networks", IEEE Trans. Power Syst. vol. 28, issue 3, pp.3047-3055, Aug. 2013.

[28] Q. Zhao, J. Garcia-Gonzalez, O. Gomis-Bellmunt, E. Prieto-Araujo, F. M. Echavarren, "Impact of converter losses on the optimal power flow solution of hybrid networks based on VSC-MTDC", Electric Power Systems Research vol. 151 pp. 395403, Oct. 2017.

[29] S. Wang, J. Guo, C. Li, S. Balasubramaniam, R. Zheng and J. Liang, "Coordination of DC power flow controllers and $\mathrm{AC} / \mathrm{DC}$ converters on optimising the delivery of wind power", IET Power Generation, vol. 10, issue 6, pp. 815-823, Jul. 2016.

[30] S. S. H. Yazdi, J. Milimonfared, S. H. Fathi, K. Rouzbehi, "Optimal placement and control variable setting of power flow controllers in multi-terminal HVDC grids for enhancing static security", Int. Journal in Elect. Power and Energy Systems, vol. 102, pp. 272-286, Nov. 2018.

[31] J. Dorn, H. Gambach, J. Strauss, T. Westerweller and J. Alligan, "Trans Bay Cable A Breakthrough of VSC Multilevel Converters in HVDC Transmission," in CIGRE Colloquium, San Francisco 2012, pp. 1-7.

[32] VSC TRANSMISSION. CIGRE Working Group B4.37, Technical Report Ref.269, 2005, April.

[33] J. Beerten, S. Cole, R. Belmans, "Generalized steady-state VSC MTDC model for sequential AC/DC power flow algorithms", IEEE Trans. Power Syst. vol. 27 issue 2. pp. 821829, May 2012. 
[34] W. Feng, A.L. Tuan, L. Tjernberg, A. Mannikoff, A. Bergman, "A new approach for benefit evaluation of multiterminal VSC-HVDC using a proposed mixed ac/dc optimal power flow", IEEE Trans. Power Deliv. vol. 29, issue 1, pp. 432443, Feb. 2014.

[35] S. Rohner, S. Bernet, M. Hiller, R. Sommer, "Modulation, losses, and semiconductor requirements of modular multilevel converters", IEEE Trans.Ind. Electron. vol. 57, issue 8, pp. 26332642, Aug. 2010.

[36] Z. Zhang, Z. Xu, Y. Xue, "Valve losses evaluation based on piecewise analytical method for MMC-HVDC links", IEEE Trans. Power Deliv. vol. 29, issue 3, pp. 13541362, Jun. 2014.

[37] U.N. Gnanarathna, A.M. Gole, A.D. Rajapakse, S.K. Chaudhary, "Loss estimation of modular multi-level converters using electro-magnetic transients simulation", in Proc. Int. Conf. Power Syst. Transient (IPST), 2011, pp. 1-6.

[38] C. Oates, C. Davidson, "A comparison of two methods of estimating losses in the Modular Multi-Level Converter", Proceedings of the 201114th European Conference on Power Electronics and Applications (EPE), Birmingham 2011, pp.110.

[39] S. Cole, "Steady-State and Dynamic Modelling of VSC HVDC Systems for Power System Simulation", Katholieke University Leuven, Belgium, Ph.D.dissertation 2010.

[40] J. Beerten, S. Cole, R. Belmans, A sequential ac/dc power flow algorithm fornetworks containing multi-terminal VSC HVDC Syst., in: Power EnergySociety General Meeting, 2010 IEEE, 2010, pp. 17, July.

[41] T. K. Vrana, S. Dennetiere, J. Jardini, Y. Yang, and H. Saad, The CIGRE B4 DC grid test system version 2013, in Electra, Mar. 2013. 


\section{List of Figures}

1 CFC topology. (a) Two currents entering and one going out. (b) Two currents going out and one entering. . . . . . . . . . . . . . . . 29

2 Average model of the two CFC structures in Fig. 1 . . . . . . . . . . . 30

3 Single phase model of one VSC station. . . . . . . . . . . . . . . . . . . 31

4 Possible connections of the CFC into the DC grid. (a) Connection CFC A. (b) Connection CFC B. (c) Connection CFC C. . . . . . . . . . . . 32

5 Scheme of the AC/DC grid used for the study. . . . . . . . . . . . . 33

6 Power flows of the AC/DC grid used for the study considering No CFC, CFC A and CFC B (powers expressed in MW) for demand 1.03. . . . . 34 


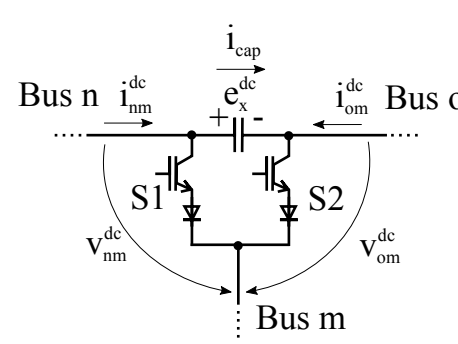

(a)

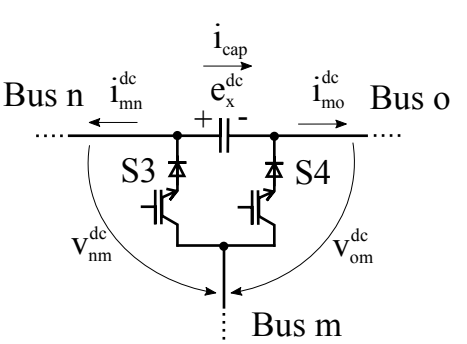

(b)

Figure 1: CFC topology. (a) Two currents entering and one going out. (b) Two currents going out and one entering. 


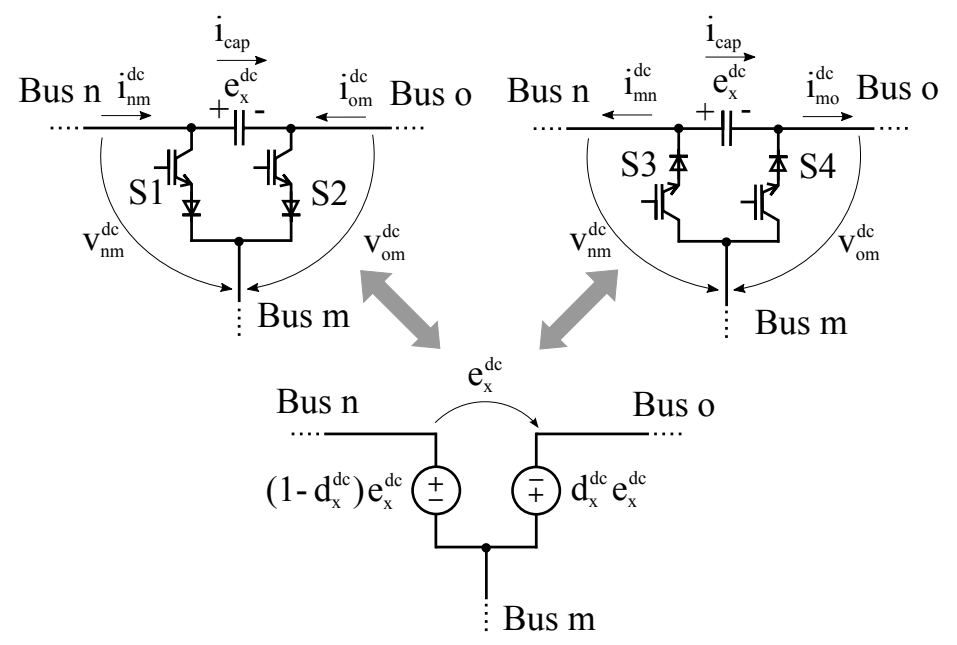

Figure 2: Average model of the two CFC structures in Fig. 1 


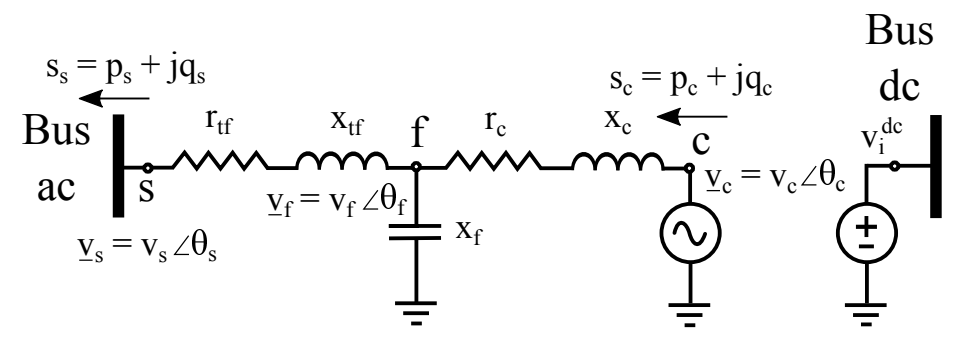

Figure 3: Single phase model of one VSC station. 


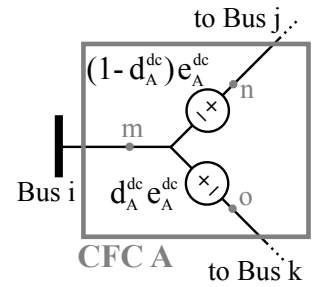

(a)

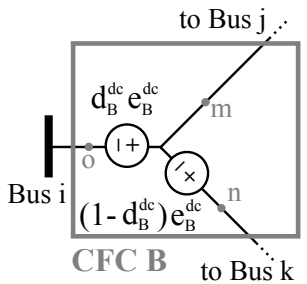

(b)

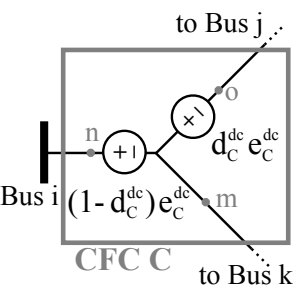

(c)

Figure 4: Possible connections of the CFC into the DC grid. (a) Connection CFC A. (b) Connection CFC B. (c) Connection CFC C. 


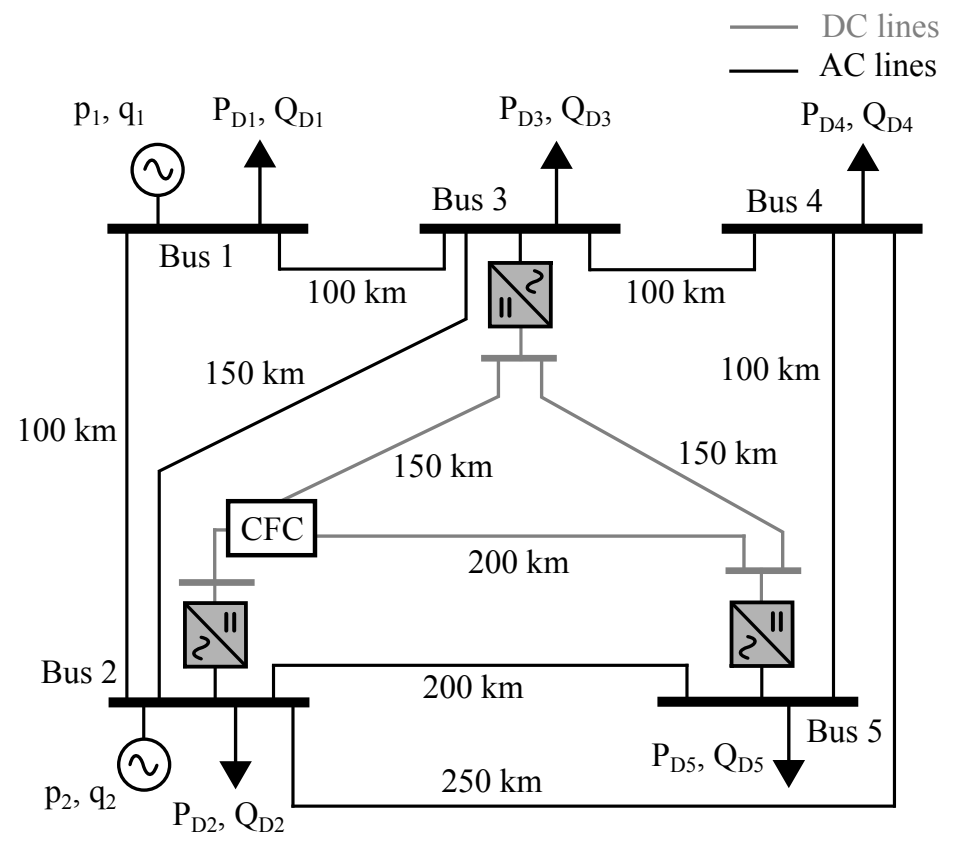

Figure 5: Scheme of the AC/DC grid used for the study. 


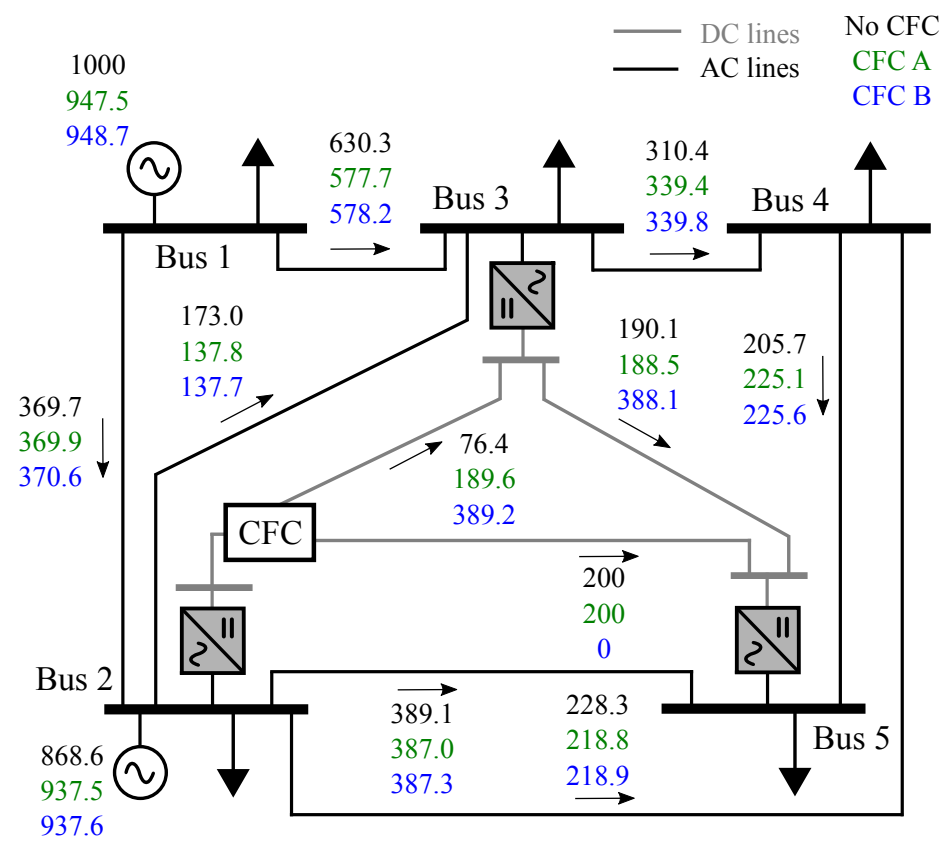

Figure 6: Power flows of the AC/DC grid used for the study considering No CFC, CFC A and CFC B (powers expressed in MW) for demand 1.03. 


\section{List of Tables}

$1 \quad$ CFC parameters . . . . . . . . . . . . . . . 36

2 Demand levels at buses for Case study 1 and $2 \ldots \ldots$. . . . . . . . . 37

3 Results Case study $1 \ldots \ldots$. . . . . . . . . . . . . . . 38

4 Results Case study $2 \ldots \ldots$. . . . . . . . . . . . . . . . . . 39 
Table 1: CFC parameters

\begin{tabular}{ccc}
\hline \hline Parameter & Symbol & Value \\
\hline Minimum CFC voltage & $\underline{E}$ & $-4 \mathrm{kV}$ \\
Maximum CFC voltage & $\bar{E}$ & $4 \mathrm{kV}$ \\
Minimum Duty cycle & $\underline{D}$ & 0 \\
Maximum Duty cycle & $\bar{D}$ & 1 \\
\hline \hline
\end{tabular}


Table 2: Demand levels at buses for Case study 1 and 2

\begin{tabular}{ccc}
\hline \hline Nominal demand & Case study 1 & Case study 2 \\
\hline$P_{D 1}$ [MW], $Q_{D 1}$ [MVAr] & 0,0 & 0,0 \\
$P_{D 2}$ [MW], $Q_{D 2}$ [MVAr] & 160,10 & 160,10 \\
$P_{D 3}$ [MW], $Q_{D 3}$ [MVAr] & 360,15 & 360,15 \\
$P_{D 4}$ [MW], $Q_{D 4}$ [MVAr] & 320,5 & 320,5 \\
$P_{D 5}$ [MW], $Q_{D 5}$ [MVAr] & 480,10 & 960,10 \\
\hline \hline
\end{tabular}


Table 3: Results Case study 1

\begin{tabular}{|c|c|c|c|c|c|c|}
\hline Demand [pu] & $\mathrm{CFC}$ & $\mathrm{OF}[€ / \mathrm{h}]$ & $p_{23}^{d c}[\mathrm{MW}]$ & $p_{25}^{d c}[\mathrm{MW}]$ & $e_{x}[\mathrm{kV}]$ & $d_{x}$ \\
\hline \multirow[t]{4}{*}{0.4} & No CFC & 16515.5 & 15.8 & 22.9 & - & - \\
\hline & CFC A & 16515.5 & 15.8 & 22.9 & $\mathbf{0}$ & 0.408 \\
\hline & CFC B & 16515.9 & 34.4 & 0 & -0.164 & 0 \\
\hline & CFC C & 16518.6 & 0 & -1.1 & -0.043 & 1 \\
\hline \multirow[t]{4}{*}{0.5} & No CFC & 21559.4 & 21.6 & 31.6 & - & - \\
\hline & CFC A & 21559.4 & 21.6 & 31.6 & 0 & 0.408 \\
\hline & CFC B & 21560.2 & 47.2 & 0 & -0.227 & 0 \\
\hline & $\mathrm{CFC} \mathrm{C}$ & 21559.6 & 0 & 47.5 & 0.141 & 1 \\
\hline \multirow[t]{4}{*}{0.6} & No CFC & 27140.1 & 27.4 & 40.3 & - & - \\
\hline & CFC A & 27140.1 & 27.4 & 40.3 & $\mathbf{0}$ & 0.405 \\
\hline & CFC B & 27141.6 & 60.3 & 0 & -0.290 & 0 \\
\hline & CFC C & 27140.6 & 0 & 60.5 & 0.180 & 1 \\
\hline \multirow[t]{4}{*}{0.7} & No CFC & 33259.9 & 33.0 & 48.7 & - & - \\
\hline & CFC A & 33259.9 & 33.0 & 48.7 & $\mathbf{0}$ & 0.404 \\
\hline & CFC B & 33265.7 & 103.8 & 0 & -0.502 & 0 \\
\hline & CFC C & 33260.6 & 0 & 73.5 & 0.219 & 1 \\
\hline \multirow[t]{4}{*}{0.8} & No CFC & 39920.8 & 38.7 & 57.2 & - & - \\
\hline & CFC A & 39920.8 & 38.7 & 57.2 & 0 & 0.403 \\
\hline & CFC B & 39924.3 & 85.4 & 0 & -0.412 & 0 \\
\hline & $\mathrm{CFC} \mathrm{C}$ & 39922.0 & 0 & 85.7 & 0.253 & 1 \\
\hline \multirow[t]{4}{*}{0.9} & No CFC & 47125.1 & 44.2 & 65.5 & - & - \\
\hline & CFC A & 47125.1 & 44.2 & 65.5 & $\mathbf{0}$ & 0.403 \\
\hline & CFC B & 47130.1 & 97.9 & 0 & -0.473 & 0 \\
\hline & CFC C & 47126.7 & 0 & 97.9 & 0.288 & 1 \\
\hline \multirow[t]{4}{*}{1.0} & No CFC & 54875.0 & 49.6 & 73.6 & - & - \\
\hline & CFC A & 54875.0 & 49.6 & 73.6 & 0 & 0.403 \\
\hline & CFC B & 54881.8 & 110.3 & 0 & -0.553 & 0 \\
\hline & CFC C & 54877.2 & 0 & 109.8 & 0.321 & 1 \\
\hline \multirow[t]{4}{*}{1.1} & No CFC & 63172.7 & 54.9 & 81.5 & - & - \\
\hline & CFC A & 63172.7 & 54.9 & 81.5 & 0 & 0.402 \\
\hline & CFC B & 63181.7 & 122.4 & 0 & -0.592 & 0 \\
\hline & CFC C & 63175.7 & 0 & 121.4 & 0.353 & 1 \\
\hline \multirow[t]{4}{*}{1.2} & No CFC & 72020.6 & 60.0 & 89.3 & - & - \\
\hline & CFC A & 72020.6 & 60.0 & 89.3 & 0 & 0.402 \\
\hline & CFC B & 72031.5 & 129.0 & 0 & -0.649 & 0 \\
\hline & $\mathrm{CFC} \mathrm{C}$ & 72024.4 & 0 & 132.7 & 0.384 & 1 \\
\hline
\end{tabular}


Table 4: Results Case study 2

\begin{tabular}{lccccccc}
\hline \hline Dem. $[\mathrm{pu}]$ & CFC & $\mathrm{OF}[€ / \mathrm{h}]$ & $p_{23}^{d c}[\mathrm{MW}]$ & $p_{25}^{d c}[\mathrm{MW}]$ & $e_{x}[\mathrm{kV}]$ & $d_{x}$ & $n p_{5}[\mathrm{MW}]$ \\
\hline 1.00 & No CFC & 88175.2 & 101.8 & 200 & - & - & 0 \\
& CFC A & $\mathbf{8 8 1 7 1 . 4}$ & $\mathbf{1 1 4 . 3}$ & $\mathbf{2 0 0}$ & $\mathbf{0 . 0 2 1} \mathbf{0 . 3 6 4}$ & $\mathbf{0}$ \\
& CFC B & 88244.2 & 311.4 & 0 & -1.639 & 0 & 0 \\
& CFC C & 88690.3 & 0 & 200 & 0.157 & 1 & 0 \\
\hline 1.01 & No CFC & 89714.0 & 85.4 & 200 & - & - & 0 \\
& CFC A & $\mathbf{8 9 6 1 0 . 4}$ & $\mathbf{1 3 6 . 1}$ & $\mathbf{2 0 0}$ & $\mathbf{0 . 1 0 3} \mathbf{0 . 4 0 5}$ & $\mathbf{0}$ \\
& CFC B & 89691.3 & 334.4 & 0 & -1.722 & 0 & 0 \\
& CFC C & 99068.5 & 0 & 200 & 0.150 & 1 & 9.6 \\
\hline 1.02 & No CFC & 94740.7 & 76.4 & 200 & - & - & 3.6 \\
& CFC A & $\mathbf{9 1 0 8 2 . 7}$ & $\mathbf{1 6 0 . 8}$ & $\mathbf{2 0 0}$ & $\mathbf{0 . 1 8 7}$ & $\mathbf{0 . 4 4 6}$ & $\mathbf{0}$ \\
& CFC B & 91172.2 & 356.9 & 0 & -1.804 & 0 & 0 \\
\hline 1.03 & CFC C & 111592.4 & 0 & 200 & 0.150 & 1 & 21.7 \\
\hline No CFC & 107271.1 & 76.4 & 200 & - & - & 15.7 \\
& CFC A & $\mathbf{9 2 6 0 1 . 4}$ & $\mathbf{1 8 9 . 6}$ & $\mathbf{2 0 0}$ & $\mathbf{0 . 2 7 1} \mathbf{0 . 4 8 7}$ & $\mathbf{0}$ \\
& CFC B & 92700.3 & 389.2 & 0 & -1.890 & 0 & 0 \\
\hline 1.04 & CFC C & 124118.6 & 0 & 200 & 0.150 & 1 & 33.8 \\
\hline No CFC & 119803.9 & 76.4 & 200 & - & - & 27.8 \\
& CFC A & $\mathbf{9 4 1 8 3 . 5}$ & $\mathbf{2 0 3 . 8}$ & $\mathbf{2 0 0}$ & $\mathbf{0 . 3 3 3} \mathbf{0 . 5 0 5}$ & $\mathbf{0}$ \\
& CFC B & 94289.8 & 401.4 & 0 & -1.953 & 0 & 0 \\
& CFC C & 136647.1 & 0 & 200 & 0.150 & 1 & 45.9 \\
\hline & No CFC & 132338.9 & 76.4 & 200 & - & - & 39.9 \\
& CFC A & $\mathbf{1 0 0 2 2 0 . 6}$ & $\mathbf{2 0 8 . 4}$ & $\mathbf{2 0 0}$ & $\mathbf{0 . 3 6 6} \mathbf{0 . 5 1 0}$ & $\mathbf{4 . 8}$ \\
& CFC B & 100614.2 & 407.5 & 0 & -1.989 & 0 & 5.1 \\
& CFC C & 149177.9 & 0 & 200 & 0.150 & 1 & 58.0 \\
\hline
\end{tabular}

